Forthcoming(2004) in Studies in History and Philosophy of Modern Physics 35(3)

\title{
Open or Closed? \\ Dirac, Heisenberg, and the Relation between Classical and Quantum Mechanics
}

\author{
Alisa Bokulich \\ Department of Philosophy, Boston University, Boston, MA 02215, USA
}

\begin{abstract}
This paper describes a long-standing, though little-known, debate between Paul Dirac and Werner Heisenberg over the nature of scientific methodology, theory change, and intertheoretic relations. Following Heisenberg's terminology, their disagreements can be summarized as a debate over whether the classical and quantum theories are "open" or "closed." A close examination of this debate sheds new light on the philosophical views of two of the great founders of quantum theory.
\end{abstract}

Keywords: Heisenberg; Dirac; Classical mechanics; Quantum mechanics; Closed theory; Intertheoretic relations

\section{Introduction}

Although Paul Dirac and Werner Heisenberg are traditionally aligned together as members of the so-called Copenhagen interpretation, when it comes to the issues of reductionism and theory change their views diverge in fundamental and interesting ways. Heisenberg and Dirac revisited their disagreements over these philosophical issues many times throughout their careers. Their disagreements, I argue, can be most succinctly described as a debate over whether physical theories are "open" or "closed." This characterization is based on Heisenberg's influential—though surprisingly little 
discussed-notion of a closed theory. The idea of a closed theory has many facets: It includes claims about the nature of scientific theories, the nature of theory change, scientific methodology, and intertheoretic relations. Heisenberg took classical and quantum mechanics both to be examples of closed theories.

A closed theory, for Heisenberg, is one that is "perfectly accurate within its domain" and "correct for all time." This notion of a closed theory had a profound impact on his understanding of theory change. Heisenberg believed that the elements of a closed theory exhibit such a tight interconnectedness that not a single concept can be changed without destroying the whole system. This means that theory change cannot happen gradually, but rather must occur all at once, by means of an "intellectual jump" that makes a "radical break" with the theories that had come before.

Although Dirac never uses the term, his own views of classical and quantum mechanics can be fruitfully understood as a rival account, namely, one of "open theories." He argues that even the most well-established parts of quantum theory are open to future revision. Instead of viewing classical mechanics as a theory that had been replaced, he saw it as a theory that should continue to be developed, modified, and extended. He took no part of physics to be a permanent achievement, correct for all time.

Dirac's view of theories as open also had an effect on his account of theory change. Where Heisenberg argued for wholesale change, Dirac sought piecemeal modifications; where Heisenberg believed that quantum theory had to make a sharp break with classical mechanics, Dirac believed that there was a deep structural continuity between these theories that should be exploited. A juxtaposition of these two thinkers not only brings us to a deeper understanding of their philosophical views, but 
also promises to shed new light on current debates over reductionism and the nature of theory change. ${ }^{1}$

\section{Heisenberg's closed theories}

In his autobiographical reflections, Heisenberg recalls having discussed his notion of a closed theory as early as 1929, during a tour of the United States that he took in part with Dirac (Heisenberg 1971, Chapter 8). Heisenberg's first published discussion of a closed theory, however, does not appear until the 1930s, and it is an idea that he revisits regularly in his writings up through the 1970s. ${ }^{2}$ Throughout these forty years his views on this topic seem to have changed very little. The German phrase that Heisenberg uses for closed theory is Abgeschlossene Theorie, where 'abgeschlossen' can be translated as 'closed,' 'locked,' 'isolated,' or 'self-contained.' Before discussing the characteristics of a closed theory, it is useful to have on hand the list of theories that Heisenberg takes to be closed.

In his book Physics and Philosophy he asserts, “One can distinguish four systems that have already attained their final form"(Heisenberg 1958, p. 98). Heisenberg identifies Newtonian mechanics as the oldest example of a closed theory. He writes, “Newton begins his Principia with a group of definitions and axioms which are interconnected in such a way that they form what one may call a 'closed system'" (Heisenberg 1958, p. 93). He continues, “The second closed system of concepts was formed in the course of the nineteenth century in connection with the theory of heat....

\footnotetext{
${ }^{1}$ In this paper I shall focus on the historical project of explicating, interpreting, and contrasting the views of Heisenberg and Dirac; the broader philosophical question of whether either of their views provides a more adequate account of the nature of theory change or of the relationship between classical and quantum mechanics (as well as an evaluation of their views against the backdrop of current philosophical scholarship on these questions) will be addressed in a future work.

${ }^{2}$ Chevalley 1988 has argued that traces of his notion of a closed theory can be found as early as 1926 (see Heisenberg 1926a, b).
} 
The third closed system of concepts and axioms has its origin in the phenomena of electricity and magnetism and has reached its final form in the first decade of the twentieth century through the work of Lorentz, Einstein and Minkowski. It comprises electrodynamics, special relativity, optics [and] magnetism" (Heisenberg 1958, pp. 9899). Last, but certainly not least, he concludes, "The fourth coherent system is essentially the quantum theory" (Heisenberg 1958, p. 100).

Heisenberg's list of closed theories immediately raises two questions: Why have these four theories been chosen and delimited in the way they have? And does he take these four to exhaust the field of physics? Heisenberg's answer to the first question is somewhat obscure and will be addressed below. His answer to the second question, however, is relatively straightforward: while these are the only four theories that currently have achieved the status of 'closed,' there are many other parts of physics that remain 'open.' For example, he believes that a fifth closed theory will probably be found someday in connection with the theory of elementary particles. Conspicuously absent from Heisenberg's list is general relativity. While special relativity is included together with electromagnetism as a single closed theory, Heisenberg believes that general relativity is still an open theory, that "has perhaps not yet achieved its final form" (Heisenberg 1958, p. 100; see also Heisenberg 1971, p. 98).

Already in his enumeration of the list, one can begin to get a sense of the features that Heisenberg takes to be characteristic of closed theories. He defines the key characteristics most concisely as follows: "By a closed theory we mean a system of axioms, definitions and laws, whereby a large field of phenomena can be described, that is mathematically represented, in a correct and noncontradictory fashion" (Heisenberg 1972, p. 123). Heisenberg was deeply impressed by axiomatic formulations of theories and took the possibility of such a formulation to be a necessary condition for a theory's 
being closed. In a 1963 interview with Thomas Kuhn for the Archive for the History of Quantum Physics (AHQP), Heisenberg traces his commitment to the importance of a consistent axiomatic formulation to David Hilbert. Heisenberg studied in Göttingen in 1922-1923, the same time Hilbert was lecturing there. ${ }^{3}$ He recalls,

I remember that in being together with young mathematicians and listening to Hilbert's lectures and so on, I heard about the difficulties of the mathematicians. There it came up for the first time that one could have axioms for a logic that was different from classical logic and still was consistent. That I think was just the essential step. ... [W] can scarcely describe nature without having something consistent, but we may be forced to describe nature by means of an axiomatic system which was thoroughly different from the old classical physics. (Heisenberg in AHQP February 25, 1963)

Heisenberg chooses the four closed theories he does because he understands each of them to consist of a distinct, closely knit group of concepts that can be given an axiomatic formulation.

The fact that a theory can be given a certain kind of axiomatic formulation, leads Heisenberg to conclude that the elements of that theory exhibit a tight interconnectedness prohibiting any further modifications or improvements. ${ }^{4}$ According to him, "the connection between the different concepts in the system is so close that one could generally not change any one of the concepts without destroying the whole system" (Heisenberg 1958, p. 94). As will be seen in the next section, this holism has strong implications for his understanding of theory development and theory change.

One of the most surprising features of Heisenberg's closed theories is his claim that they are perfectly accurate within their domain and correct for all time. In his 1948 Dialectica article, he explains "What, then, finally, is the truth content of a closed-off theory? ... The closed-off theory holds for all time; wherever experience can be

\footnotetext{
${ }^{3}$ For a more thorough discussion of the influence of Hilbert on Heisenberg see Frappier (forthcoming).
} 
described by means of the concepts of this theory, even in the most distant future, the laws of this theory will always prove correct" (Heisenberg 1948, p. 45). There are two components to this claim: first, that a closed theory is an accurate description of a certain domain of phenomena, and second, that it is a final description of a particular domain of phenomena-a permanent achievement that cannot be called into question by any future developments of science.

To understand these claims of accuracy and finality it is helpful to examine how Heisenberg applies them to a particular closed theory such as classical mechanics. He explains, "Newtonian mechanics is a limited description of nature and in that limited field it is perfectly accurate. All attempts to improve Newtonian mechanics are just fruitless. But you come to parts of physics where the concepts of Newtonian physics don't apply. You just can't do anything with these words. Then you have to go for something new" (Heisenberg in AHQP, February 27, 1963). The important qualification on the "perfectly accurate" claim is the restriction of the domain of applicability of the theory. Classical mechanics thus, while true, is not a universal theory; there are other domains of phenomena that must be explained by means of different closed theories. On the question of how this domain of applicability is to be delimited, Heisenberg's answer is unsatisfactory: the domain of classical mechanics is determined by where the concepts of classical mechanics can be applied. He writes, "Wherever concepts like mass, velocity and force can be applied unhesitatingly, there Newton's law, that the force is equal to the product of mass and acceleration, will be shown to be true" (Heisenberg 1935 [1979], pp. 41-42). He does not, however, offer us any independent way of determining when it is that the concepts of classical mechanics can be applied.

\footnotetext{
${ }^{4}$ Unfortunately Heisenberg does not seem to have offered a detailed argument or justification for this
} conclusion. 
Coupled to the claim that closed theories are accurate is his claim that they are also final: "Newtonian mechanics cannot be improved in any way, for inasmuch as we can describe a particular phenomenon with the concepts of Newtonian physics ... Newton's laws hold quite rigorously, and nothing in this will be changed for the next hundred thousand years. ... Newtonian mechanics is fully valid now and will remain so in the future" (Heisenberg 1971, p. 96). Heisenberg offers two sorts of justifications for his claim that closed theories, such as Newtonian mechanics, are correct and unable to be improved. The first is historical: he claims that even the oldest of the closed theories, classical mechanics, has never been improved. ${ }^{5}$ To the obvious objection that quantum mechanics is an improvement on classical mechanics, Heisenberg responds that this shift was a matter of radical reconstruction, and not minor improvements or modifications. The second—and in his mind stronger-argument for the finality of closed theories rests on what he calls "their compactness and manifold confirmation by experiment" (Heisenberg 1972, p. 124). By compactness, he means that a wide range of phenomena can be accounted for by means of a small number of axioms, postulates and so on. Finality is not, however, a purely logical feature of closed theories: Heisenberg also understands it to be a claim that is justified empirically. Closed theories have not only withstood the test of time, but they have been confirmed by a wide range of phenomena.

The picture that starts to emerge from Heisenberg's account of closed theories is a kind of theoretical pluralism: contemporary science is characterized by a handful of distinct closed theories, each with its own circumscribed domain, within which the theory is perfectly accurate and unalterable.

\footnotetext{
${ }^{5}$ For a contrasting view see Dirac 1951, p. 10 quoted below.
} 


\section{Heisenberg's methodology and model of theory change}

Heisenberg's philosophy of closed theories is also reflected in his scientific methodology. One well-known aspect of his methodology is his attempt to construct new theories by restricting himself to observable quantities. In 1926 he declares that the root of the difficulties facing the development of quantum theory is

the inappropriate transfer of classical concepts and ideas to the problems of atomic structure.... The program of quantum mechanics must therefore be first of all to free itself from these intuitive [anschaulichen] pictures, and, in place of the laws of classical kinematics and mechanics used up until now, to establish simple relations between experimentally given quantities. (Heisenberg 1926b, p. 990)

Heisenberg describes this approach as being the one that guided not only his development of matrix mechanics, but also his attempts in the early 1940s to construct an S-matrix alternative to quantum field theory. ${ }^{6}$ As James Cushing notes in his book on the history of the S-matrix, "Heisenberg wanted to avoid any reference to a Hamiltonian or to an equation of motion and to base his theory only on observable quantities" (Cushing 1990, p. 33). As will be shown, this approach is in direct contrast with Dirac's view that a Hamiltonian formulation and equations of motion are of paramount importance. In the above quotation from Heisenberg, we see yet another aspect of his methodology that stands in opposition to Dirac's: namely, his view that in the development of quantum theory one should not make use of classical mechanics.

The holistic and interconnected nature of a closed theory means, for Heisenberg, that the solution to a scientific problem can never be achieved by modifying just one element of the theory. In another interview with Kuhn, where he is discussing the

\footnotetext{
${ }^{6}$ There is, of course, the very important question of whether Heisenberg's self-understanding of his methodology accurately reflects his scientific practice. Chevalley (1988), for example, who also discusses this passage, notes in passing that it is not at all clear that this is the methodology Heisenberg is in fact using in 1925 and 1926. The question of whether these actors' methodological pronouncements are in conformity with their practice is not a question that I shall pursue here.
} 
development of quantum electrodynamics (QED), Heisenberg notes how his approach was fundamentally different from Dirac's:

Dirac and I had always been rather opposite to each other in this question of how to attack problems. I would always look for the connections. I would say, 'I can never solve one difficulty at a time. I can solve only 100 difficulties at the time, or I can never do anything. Everything is connected to everything, and the whole thing must give a consistent picture.' But Dirac would say, 'No, that is impossible because nobody is so clever as to solve 100 difficulties at a time; you have to concentrate on one point. (Heisenberg in AHQP, February 28, 1963)

Whereas for Dirac, theory development could proceed piecemeal by means of gradual modifications, for Heisenberg it must take place all at once. How then does Heisenberg deal with the discovery of new phenomena that cannot be adequately accounted for within the framework of an existing theory? In his 1934 discussion of closed theories his answer is clear: "the transition in science from previously investigated fields of experience to new ones will never consist simply of the application of already known laws to these new fields. On the contrary, a really new field of experience will always lead to the crystallization of a new system of scientific concepts and laws" (Heisenberg 1934 [1979], p. 24). In other words, new phenomena require the formation of a new closed theory.

In his recollections of a 1929 conversation with Barton Hoag (an experimental physicist at Chicago) Heisenberg explains how this pattern describes the development from Newtonian mechanics to quantum theory. A recurring theme in Heisenberg's discussion is how progress in science is fundamentally different from progress in engineering. He writes,

To my mind, it is fundamentally wrong to compare the basic changes involved in the transition from Newtonian mechanics to relativistic or quantum mechanics with the improvements of the engineer.... Newtonian physics constitutes a closed system. ... It is thanks to this coherence that there can be no minor improvements. All we can do is to adopt an entirely new conceptual system. (Heisenberg 1971, p. 97) 
At this point Heisenberg recalls Hoag interjecting with the following question: "Why do you lay so much stress on the fact that the transition from one realm to another, for instance from Newtonian physics to quantum theory, is not continuous but discrete?" Heisenberg dismissively identifies the view that development in science is continuous, with the pragmatism of the engineer: "Your idea of continuous progress as we know it from engineering would weaken, or rather soften, physics to such an extent that we could hardly continue to call it an exact science" (Heisenberg 1971, p. 98). ${ }^{7}$

After Heisenberg and Dirac meet up in Chicago in 1929, the two decided to return to Europe together by a way of ship from San Francisco to Yokohama. During this long sea voyage together, Heisenberg recalls discovering that Dirac held this pragmatic and gradualist view opposite to his own. Heisenberg describes Dirac as someone who "felt that the development of our science was a more or less continuous process.... For once you use the pragmatic approach, you are bound to consider the progress of science as a continuous and never-ending process" (Heisenberg 1971, p. 101). For Heisenberg the gradualist model of theory change is unacceptable because it threatens the integrity of physics as an exact science. He believes that only by having a closed theory could one legitimately speak of physical laws and be assured a consistent, meaningful description of nature.

\section{Closed theories and intertheoretic relations}

From the preceding discussion one might have noticed that Heisenberg's holist model of theory change bears many striking similarities to Kuhn's model of scientific revolutions. In her book, Quantum Dialogue, Mara Beller draws out some these similarities between Heisenberg's closed theory and Kuhn's paradigm. She notes,

\footnotetext{
${ }^{7}$ For a contrasting view see Dirac in AHQP, April 1, 1962, quoted below.
} 
"Both resist improvement by small changes. Both are holistic. Both demand that advances come by way of jumps" (Beller 1999, p. 290). Interestingly this is a similarity that did not escape the notice of either Heisenberg or Kuhn. Heisenberg had seen a copy of the first edition of The Structure of Scientific Revolutions shortly before Kuhn's interview of him for the Archive for the History of Quantum Physics, and the question of the similarity between their ideas came up during the course of the interview. In this interview Heisenberg remarks, "I have studied your book already and it gave me great pleasure to see the way how you use the term paradigm.... Yes, the necessity is to break away.... One always, in such a situation, is forced to cut the branch on which one is sitting" (AHQP February 27, 1963).

Another point of similarity between their views is that in Heisenberg's writings one comes across claims that sound like a formulation of the incommensurability thesis (though he does not use this term). For example, in response to a question regarding the relationship between sets of concepts belonging to different closed theories, he poses a further question:

If, for instance, the same concepts or words occur in two different sets and are defined differently with regard to their connection and mathematical representation, in what sense do the concepts represent reality? The concepts of space and time belonged both to Newtonian mechanics and to the theory of relativity. But space and time in Newtonian mechanics were independent; in the theory of relativity they were connected. (Heisenberg 1958, pp. 97-98).

Heisenberg brings up a similar point again in his interview with Kuhn:

If an experiment does not fit in Newtonian physics, you don't know what you mean by the words. That is the point. As soon as you come to velocities, near the velocity of light, then it is not only so that Newtonian physics does not apply, but the point is that you even don't know what you mean by 'velocity.' ... That, I think is a very characteristic feature of what I mean by close[d] system; that is, when you have such a system and you get disagreement with the facts, then it means that you can't use the words anymore. You just don't know how to talk. (Heisenberg in AHQP, February 27, 1963) 
Kuhn's immediate response in this interview is to say “O. K. That I entirely agree with. But I think you look too narrowly at the group of systems within which one can have that experience" (Kuhn in AHQP, February 27, 1963). For example, while Kuhn would argue that Ptolemaic astronomy also has this feature, Heisenberg would not want to count the Ptolemaic system as an example of a closed theory. ${ }^{8}$

Despite these similarities, there are a number of points on which their views diverge. One striking difference is that, while Heisenberg's closed theories are seen to be a permanent achievement, Kuhn's paradigms are best understood in terms of wholesale theory replacement. In this same interview Kuhn explains to Heisenberg, "As you perhaps see in the book, I am not very happy about taking out a sub-group of these important discarded theories and saying, 'Now these few are still with us.'. . In the long run quantum mechanics must be the substitute for Newton in the sense that Newton is the substitute for Ptolemy and Aristotle" (Kuhn in AHQP, February 27, 1963). In this sense Kuhn—unlike Heisenberg—is a serial monogamist, not a theoretical pluralist.

Another important issue that divides Kuhn and Heisenberg is realism. As was discussed earlier, Heisenberg rejects a pragmatic interpretation of physical theories as approximate descriptions of the phenomena. Furthermore he takes the laws within closed theories to be correct for all time. One can see Heisenberg attempting to articulate his realist views in quotations such as the following:

This whole subject is extremely difficult to put into words. If, as we must always do as a first step in theoretical physics, we combine the results of experiments and formulae and arrive at a phenomenological description of the processes involved, we gain the impression that we have invented the formulae ourselves. If, however, we chance upon one of those very simple, wide relationships that must later be incorporated into the axiom system ... then we

\footnotetext{
${ }^{8}$ For a more complete discussion of the similarities and dissimilarities between Heisenberg and Kuhn, see Bokulich (forthcoming).
} 
are quite suddenly brought face to face with a relationship that has always existed, and that was quite obviously not invented by us or by anyone else. Such relationships are probably the real content of our science. (Heisenberg 1971, p. 99)

Heisenberg's view thus seems to be a theoretical pluralism combined with realism, though it is not clear how he intends to coherently combine these views. ${ }^{9}$

Heisenberg's philosophy of closed theories has strong implications for his views on intertheory relations in general, and the relationship between classical and quantum mechanics in particular. He repeatedly maintains that quantum mechanics is neither a falsification of classical mechanics nor an improvement upon it. When it comes to saying precisely what is the proper way to understand the relationship between classical and quantum mechanics, Heisenberg merely falls back upon Niels Bohr's indispensability of classical concepts: "Newtonian mechanics is a kind of a priori for quantum theory. It is a priori in the sense that it is the language which enables us to say what we observe" (Heisenberg in AHQP February 27, 1963). It is not clear, however, that this Bohrian line can be made consistent with Heisenberg's view that closed theories are "complete in themselves." ${ }^{10}$

Heisenberg's 1934 comment regarding the issue of intertheoretic relations seems truer to his mark: "The edifice of exact science can hardly be looked upon as a consistent and coherent unit in the naive way we had hoped (Heisenberg 1934 [1979], p. 25). By committing himself to the picture of theories as largely isolated, closed

\footnotetext{
${ }^{9}$ One possible interpretation would be along the lines of Nancy Cartwright's "metaphysical nomological pluralism" described in Cartwright 1995 and 1999. Hans Radder (personal communication) has suggested that referential realism may be another way in which to interpret Heisenberg's views. One of the few places in which Heisenberg attempts to elaborate his pluralistic realism is in his unpublished manuscript of 1942. A fuller examination of these metaphysical issues, which fall outside the scope of his debate with Dirac, will be pursued in a future work.

${ }^{10}$ As Beller (1996) notes, "Heisenberg and Born often supported Bohr's position, although it was incompatible with their own. Such public support is one of the major sources of contradictions in Heisenberg's and Born's writings"' (p. 184).
} 
axiomatic systems, Heisenberg has left little room for a substantive theory of intertheoretic relations. ${ }^{11}$

\section{Dirac's open theories}

Whereas Heisenberg interpreted classical and quantum mechanics as closed theories, perfectly accurate within their domains, unable to be altered, and correct for all time, Dirac saw these theories as open: only approximate, able to be modified piecemeal, and having no parts immune to future revision. In a 1962 interview by Kuhn we see Dirac embracing the idea that progress in physics is like progress in engineering:

I think this engineering education has influenced me very much in making me learn to tolerate approximations.... I learned that even a theory based on approximations could be a beautiful theory. I rather got to the idea that everything in nature was only approximate, and that one had to be satisfied with approximations, and that science would develop through getting continually more and more accurate approximations, but would never attain complete exactness. ... As a result of that, I haven't been much interested in questions of mathematical logic.... I feel that these things are just not important, that the study of nature through getting ever improving approximations is the profitable line of procedure. (Dirac in AHQP, April 1, 1962)

There are a number of key points that emerge from these comments by Dirac. First, that Dirac's oft-quoted insistence on "beautiful theories" should not be interpreted as an insistence on perfectly accurate or exact theories. ${ }^{12}$ Second, we see Dirac advocating a gradualist model of theory change, whereby progress in physics takes place through continually developing ever more accurate approximations. Third, Dirac seems rather dismissive of the ideals of mathematical logic, which had made such an impression on Heisenberg.

\footnotetext{
${ }^{11}$ Scheibe (2001) also notes the threat that Heisenberg's closed theories pose for the unity of science.

${ }^{12}$ In Section 7 I will return to a discussion of what Dirac means by "beauty."
} 
For Dirac neither quantum mechanics—nor classical mechanics—has reached its final form. Unlike Heisenberg, who took classical mechanics to be essentially unchanged since the opening pages of the Principia were written, Dirac sees classical mechanics as a theory that has been modified and improved: "Classical mechanics is essentially the mechanics of Newton. This mechanics was very much developed by Lagrange, Hamilton, and others, and more recently it has been modified in its underlying ideas through the appearance of Relativity" (Dirac 1951, p. 10). While Heisenberg took classical mechanics and relativity to be two distinct closed theories, for Dirac they belong to the same theory. ${ }^{13}$ Similarly Dirac criticized the Copenhagen theorists for claiming that quantum theory had attained its final form. In a 1929 letter to Bohr he writes,

I am afraid I do not completely agree with your views. Although I believe that quantum mechanics has its limitations and will ultimately be replaced by something better, ... I cannot see any reason for thinking that quantum mechanics has already reached the limit of its development. I think it will undergo a number of small changes. (Dirac's letter to Bohr, Dec. 9, 1929, in AHQP $)^{14}$

In this quotation we not only see Dirac's commitment to the open character of quantum theory, but also his endorsement of a gradualist model of theory change. As will be shown next, Dirac's view that classical and quantum mechanics are both open theories is the cornerstone of his scientific methodology.

\section{Dirac's methodology and model of theory change}

In his own recollections about the early development of quantum theory Dirac also notes some of the methodological differences between his approach and

\footnotetext{
${ }^{13}$ In addition to relativity, Dirac also includes classical electrodynamics in with classical mechanics. These are then contrasted with quantum mechanics (which includes quantum electrodynamics).

${ }^{14}$ Quoted in Kragh 1990, p. 92.
} 
Heisenberg's: "If you look up these early papers you will see that there is quite a difference in our styles, because in my work the noncommutation was the dominant idea. With the Göttingen School, the dominant idea was the use of quantities closely connected with experimental results and the noncommutation appeared as secondary and derived" (Dirac 1971, p. 30). Recall that in 1925 Dirac showed that the "Heisenberg product," (or what we now—following Dirac's terminology—call the commutator) that appears in Heisenberg's matrix mechanics paper is equal to it times the classical Poisson bracket. In this paper, Dirac remarks "the correspondence between the quantum and classical theories lies not so much in the limiting agreement when $h \rightarrow 0$ as in the fact that the mathematical operators in the two theories obey in many cases the same laws" (Dirac 1925, p. 649). ${ }^{15}$ Dirac took correspondences such as this to indicate a deep structural similarity between classical and quantum mechanics—a similarity that could be exploited in the further development of these theories. One might refer to this aspect of Dirac's philosophy as the thesis of structural continuity.

In his well-known textbook The Principles of Quantum Mechanics Dirac again emphasizes this continuity: "We should ... expect to find that important concepts in classical mechanics correspond to important concepts in quantum mechanics, and, from an understanding of the general nature of the analogy between classical and quantum mechanics, we may hope to get laws and theories in quantum mechanics appearing as simple generalizations of well-known results in classical mechanics" (Dirac 1958, p. 84).

The importance that Dirac places on the analogy between classical and quantum mechanics can help us make sense of the dismissive attitude that he took towards Schrödinger's formulation of quantum theory. It is often thought that this critical stance

\footnotetext{
${ }^{15}$ I will return to discuss Dirac's view on the correspondence between classical and quantum mechanics more fully in Section 7.
} 
towards Schrödinger was a product of some sort of allegiance on the part of Dirac to the Copenhagen-Göttingen School. Instead, I would argue that Dirac's preference for the Heisenberg formulation is due to the fact that it makes explicit the structural similarities between the classical and quantum formalisms. Although Dirac is of course aware of the equivalence of these two formulations and notes the practical utility of Schrödinger's formulation, he maintains, "Heisenberg's form for the equations of motion is of value in providing an immediate analogy with classical mechanics and enabling us to see how various features of classical theory ... are translated into quantum theory" (Dirac 1958, pp. 115-116).

One might wonder why Dirac thought that it was so important to make this analogy explicit. The reason, I argue, is that Dirac saw in this analogy an important tool for the further development of scientific theories. In a 1945 paper called "On the Analogy Between Classical and Quantum Mechanics" Dirac begins,

There are two forms in which quantum mechanics can be expressed, based on Heisenberg's matrices and Schrödinger's wave functions respectively.... The first is in close analogy with classical mechanics, as it may be obtained from classical mechanics simply by making the variables of classical mechanics into non-commuting quantities satisfying the correct commutation relations. The development of the analogy has been greatly hampered by the mathematical methods available for working with non-commuting quantities.... The present paper will show how ... in the case when the non-commuting quantities are observables, one can set up a theory of functions of them ... [and] make closer the analogy between classical and quantum mechanics. (Dirac 1945, p. 195)

In this paper Dirac further attempts to develop a theory that allows one to assign a probability for non-commuting observables to have definite values, though he notes that this probability—being a complex number—cannot be given a physical interpretation. Perhaps surprisingly, he then goes on to show how this formal probability can be used "to set up a quantum picture rather close to the classical picture in which the coordinates $q$ of a dynamical system have definite values at any time" 
(Dirac 1945, p. 197). In other words, Dirac develops a way to introduce particle trajectories into quantum mechanics-something that he takes to be an important extension of the analogy between classical and quantum mechanics.

The picture that begins to emerge here is what might be called a methodology of analogy extension. Dirac sees between classical and quantum mechanics certain formal or mathematical similarities that he thinks can be fruitfully extended in new directions. I want to argue that the way in which Dirac goes about extending this analogy can itself be given a more precise characterization in terms of what I call the reciprocal correspondence principle. The reciprocal correspondence principle can be understood as the interplay between the following two components: First, the standard correspondence principle, which I take here to mean quite broadly the use of classical mechanics in the further development of quantum theory. ${ }^{16}$ And, second, what José Sánchez-Ron (1983)—in connection with the work of Adriaan Fokker-has called the inverse correspondence principle, by which one uses problems in quantum theory to guide the further development of classical mechanics. ${ }^{17}$

In a rare moment of methodological reflection in a lecture at the 1949 Canadian Mathematical Congress, Dirac provides a clear account of what I have here called the reciprocal correspondence principle. He explains,

My own opinion is that we ought to search for a way of making fundamental changes not only in our present Quantum Mechanics, but actually in Classical Mechanics as well. Since Classical Mechanics and Quantum Mechanics are closely connected, I believe we may still learn from a further study of Classical

\footnotetext{
${ }^{16}$ One must be cautious in identifying this generic understanding of the correspondence principle with Bohr's more specific views. I would further argue that because of their very different approaches to intertheoretic relations, neither Heisenberg nor Dirac really shared Bohr's understanding of the correspondence principle. The relation of Bohr's views to the two thinkers considered here will be examined in a future work.

${ }^{17}$ In a footnote, Sánchez-Ron notes that the inverse correspondence principle methodology could be applied to Dirac's work as well. Helge Kragh, in his excellent biography of Dirac, also notes the similarities between Fokker's methodology and Dirac's, though in this connection the wrong paper by Sánchez-Ron is referenced.
} 
Mechanics. In this point of view I differ from some theoretical physicists, in particular Bohr and Pauli. (Dirac 1951, p. 18)

Although Dirac does not explicitly mention Heisenberg here, it is likely that he also has him in mind. In this quotation we see once again that Dirac not only takes quantum mechanics to be an open theory, capable of further modification, but that he takes classical mechanics to be open as well.

The year in which Dirac is presenting this lecture (1949) suggests that he is concerned with the recent developments in quantum electrodynamics and quantum field theory. In particular, Dirac was notoriously unsatisfied with the divergencies or infinities plaguing quantum electrodynamics and with those who attempted to fix these problems with renormalization methods.

To see more clearly how different Dirac's methodology is from Heisenberg's and the rest of the Copenhagen-Göttingen group it is worth quoting a bit further from this lecture. He begins, "It seems to me that one ought to separate the difficulties as far as possible ... and attack each one separately." Notice the difference here from Heisenberg who thought a hundred problems needed to be solved at once. Dirac continues,

For the purpose of this attack, we may very well work with the concepts of Classical Mechanics.... We want to find new ideas and I think it is very likely that some of the new ideas which we are looking for could be expressed and understood simply on the basis of Classical Mechanics. ... In this respect I differ from Pauli, who believes that Classical Mechanics has nothing more to teach us in the Quantum Theory, and that to make advances one should work with the most highly developed form of our present Quantum Mechanics.... I believe that working with a highly developed form, which is necessarily a complicated form, restricts one's power for getting new ideas, and if there are new ideas which can be understood on a Classical basis, then one should try to work them out simply keeping to the Classical Theory, and after one has worked them out one can transfer them to the Quantum Theory by using the already established connection between Classical Mechanics and Quantum Mechanics.

(Dirac 1951, p. 20) 
In this last line we see another important component of Dirac's methodology, which we may call the thesis of transferability. According to this thesis, any progress that we are able to make within the classical context will subsequently be transferable back into the quantum context.

It should be noted that Dirac did not see these new required advances as being confined to the so-called borderlands of our theories. Rather, he thought they could reach to the very heartland. He says, "The new ideas which I believe we should try to get may necessitate a fundamental change even in our description of those phenomena which can be quite well described by the existing quantum mechanics. ... For this reason, I think that one should examine closely even the elementary and the satisfactory features of our present Quantum Mechanics"' (Dirac 1951, pp. 20-21). In other words, the open character of classical and quantum mechanics meant for Dirac that there was no part of these theories that was an achievement, correct for all time, as Heisenberg would say.

One of the clearest examples of an application of the reciprocal correspondence principle is found in Dirac's 1938 paper "Classical Theory of Radiating Electrons." ${ }^{18}$ In this work he writes,

If we accept Maxwell's theory, the field in the immediate neighborhood of the electron has an infinite mass. This difficulty has recently received much prominence in quantum mechanics ... where it appears as a divergence in the solution of the equations that describe the interaction of an electron with an electromagnetic field.... One may think that this difficulty will be solved only by a better understanding of the structure of the electron according to quantum laws. However, it seems more reasonable to suppose that the electron is too simple a thing for the question of the laws governing its structure to arise, and thus quantum mechanics should not be needed for the solution of the difficulty. Some new physical idea is now required, an idea which should be intelligible both in the classical theory and in the quantum theory, and our easiest path of approach to it is to keep within the confines of the classical theory. (p. 149)

\footnotetext{
${ }^{18}$ I thank Matthias Frisch, who discusses the nonlocal character of the theory that Dirac develops here (Frisch 2002), for bringing this paper to my attention.
} 
In this quotation we clearly see the reciprocal correspondence principle at work. As a way of solving the problem of divergencies in quantum electrodynamics, Dirac turns to the development of a more adequate classical electrodynamics. Dirac's thesis of structural continuity-in this case between the classical and quantum theories of the electron-undergirds his thesis of transferability, which means that the classical solution to the problem of divergences that he obtains here will be transferable back to the quantum theory. One of the justifications he offers for this methodology is the pragmatic point that the classical theory provides a simpler context in which to work out new ideas.

Dirac never abandoned the view that classical and quantum mechanics were open theories. Twenty-five years after his paper on the classical electron, he was still making use of the reciprocal correspondence principle. Although Dirac never accepted renormalization theory, he turned his attention to the problems that renormalization theory left unsolved. Among these he lists the following:

One of the problems is ... accounting for the number 137. Other problems are how to introduce the fundamental length to physics in some natural way, how to explain the ratios of the masses of the elementary particles and how to explain their other properties. I believe separate ideas will be needed to solve these distinct problems and that they will be solved one at a time through successive stages in the future evolution of physics. At this point I find myself in disagreement with most physicists. They are inclined to think one master idea will be discovered that will solve all these problems together. (Dirac 1963, p. 50)

Clearly Heisenberg would be counted among those who believed these various problems needed to be solved all at once.

One of Dirac's more surprising approaches to solving these problems involved reintroducing an aether. Once again, he took the key to solving a quantum problem to lie in the development of a more adequate classical theory. In 1951 he had developed yet another classical electrodynamics, one that required postulating a velocity field 
defined at all points of space-time. Dirac interpreted this velocity as the velocity of the aether relative to the Earth. He argued that such an aether could be rendered consistent with relativity theory as long as one subjected the aether velocity to the quantum uncertainty relations. In this way Dirac was able to recover the Lorentz invariance of his theory. When, in 1952, Leopold Infeld pointed out that one could accept all of the conclusions of Dirac's new electrodynamics without postulating an aether, Dirac responded as follows: "Infeld has shown how the field equations of my new electrodynamics can be written so as not to require an aether. This is not sufficient to make a complete dynamical theory. It is necessary to set up an action principle and to get a Hamiltonian formulation of the equations suitable for quantization purposes, and for this the aether velocity is required" (Dirac 1952). For Dirac, the Poisson bracket correspondence that he had discovered in 1925 provided an important link between classical and quantum mechanics. One can only take an advantage of this correspondence if one has a Hamiltonian version of the classical theory. Thus in his search for a new QED, his strategy was to develop an appropriate Hamiltonian version of classical electrodynamics, which could then be quantized. If this meant reintroducing an aether and absolute simultaneity, then he was willing to do this. This reinforces the fact that, for Dirac, even the most accepted and well-established parts of theories were open to future revision.

When confronted with these same difficulties of QED, Heisenberg, by contrast, attempted to solve all of these problems at once by restricting himself to observables only-the same trick that had worked for him in 1925. This approach led Heisenberg to abandon quantum field theory in favor of the S-matrix program. For Dirac, on the other hand, agreement with experiments was not the final test of a theory. Regarding renormalization theory he writes, "Just because the results happen to be in agreement 
with observation does not prove that one's theory is correct" (Dirac 1987, p. 196). Dirac was quite critical of both renormalization and S-matrix theory. Despite the progress of the S-matrix school, he believed that high-energy physics should be based instead on equations of motion (see, for example, Dirac 1970, p. 30). As I will show in the next section, one can only make sense of Dirac's insistence on equations of motion in the context of his fundamental belief in the unity of physics.

\section{Dirac and intertheoretic relations}

Dirac's views on intertheoretic relations do not fit easily into the usual categories of either reductionism or pluralism. While he clearly thinks that there is a close relationship between classical and quantum mechanics, he denies that this relationship is captured by the usual reductionist picture that classical mechanics is recovered from quantum mechanics in the limit $\hbar \rightarrow 0$. As was mentioned above, he states in his seminal 1925 paper that "the correspondence between the quantum and classical theories lies not so much in the limiting agreement when $\hbar \rightarrow 0$ as in the fact that the mathematical operators in the two theories obey in many cases the same laws" (Dirac 1925, p. 649). It is not clear, however, what exactly Dirac means when he says that the mathematical operators obey the same laws. On the most superficial reading, Dirac is merely pointing to the formal similarity between the classical Poisson bracket relation and the quantum commutator. Olivier Darrigol, however, has suggested that in this passage Dirac is making a more fundamental point: "The correspondence between two theories, he [Dirac] believed, was not limited to the form of the fundamental equations; it concerned mathematical structures, in the modern sense of the word" (Darrigol 1992, p. 317). While Darrigol is quite right in pointing this out, the precise nature of Dirac's structural approach to intertheoretic relations is in need of further investigation. 
What is clear is that Dirac is a firm defender of the unity of physics. In a 1970 paper we see Dirac linking three key themes together with his quest for the unity of physics: the relative unimportance of agreement with experiment, the necessity of equations of motion, and mathematical beauty. He writes,

If we believe in the unity of physics, we should believe that the same basic ideas universally apply to all fields of physics. Should we not then use the equations of motion in high-energy as well as low-energy physics? I say we should. A theory with mathematical beauty is more likely to be correct than an ugly one that fits some experimental data. (Dirac 1970, p. 29)

There are two points in this quotation that require further clarification: The first is the question of why Dirac places so much emphasis on equations of motion. The answer can be found little further on in this same paper. He explains,

High-energy physics forms only a small fraction of the whole of physics. The theories of most fields, such as solid-state physics, spectroscopy of atoms and molecules, and chemical physics, are based, fairly satisfactorily, on equations of motion. We believe in the unity of physics. The equations of motion that are so successful for most of physics cannot be simply discarded for one branch of physics. Although these equations may need modification, perhaps involving different kinds of variables, one would still expect to retain the basic structure. (Dirac 1970, p. 30)

Thus, Dirac's commitment to equations of motion can be understood in terms of his more fundamental belief in the unity of physics. A branch of physics formulated without equations of motion will, in his view, remain disconnected from the rest of physics. While such a disconnect might be expected by those who believe that the various branches of physics should be closed theories that are complete-in-themselves, for an advocate of open theories, there remains the hope that these various branches can be unified. 
The second point in need of clarification is the perennial question of what Dirac means by mathematical beauty. ${ }^{19}$ While the notion of beauty is notoriously vague and multifaceted, I want to argue that one often-overlooked component of what Dirac means by beauty is a continuity and structural similarity with classical mechanics. This aspect of his understanding of beauty is expressed most clearly in a 1927 unpublished manuscript where he remarks,

The quantum theory has now reached a form ... in which it is as beautiful, and in certain respects more beautiful than the classical theory. This has been brought about by the fact that the new quantum theory requires very few changes from the classical theory, these changes being of a fundamental nature, so that many features of the classical theory to which it owes its attractiveness can be taken over unchanged into the quantum theory" (Dirac 1927, AHQP, emphasis added; quoted in Darrigol 1992, p. 345).

For Dirac, classical mechanics is a beautiful theory that remains a benchmark against which all other physical theories are to be measured. Beginning with his work in 1925, he saw hints of a structural continuity between classical mechanics and the new quantum theory. It was his belief in the open nature of these theories that led him to devote his life to trying to extend and deepen these structural similarities in new ways.

\section{Conclusion}

In this historical debate between Heisenberg and Dirac there is a marked richness in thinking about the nature of scientific methodology, theory change, and intertheoretic relations. Many of the arguments they raise mirror our contemporary philosophical debates over these same issues. On the question of theory change we can recognize in their writings the familiar positions of the gradualist and the holist: Can theories be modified piecemeal, or must we opt for wholesale exchange? Similarly,

\footnotetext{
${ }^{19}$ For a discussion of the role of beauty in science, including a discussion of Dirac's commitment to beauty, see McAllister 1996.
} 
when it comes to the question of methodology, we see Heisenberg and Dirac advocating two very different accounts of heuristics: Should theory development progress by focusing on experimentally given quantities and make a break with what came before, or should new theories be developed through analogy extension, retaining those elements that were successful in predecessor theories?

One example of a current debate that can be significantly enriched by this historical material is the debate over intertheoretic relations in general, and the relationship between classical and quantum mechanics in particular. Current accounts of the relationship between these two theories typically assume either a replacement view (quantum mechanics replaced classical mechanics rendering it obsolete) or a reductionist view (quantum mechanics reduces to classical mechanics under a limiting procedure). What is particularly interesting about this historical debate is that neither Heisenberg nor Dirac adopt either of these views.

Both Heisenberg and Dirac argue for the continued importance of classical mechanics, though as we have seen, they do so in quite different ways. For Heisenberg, classical mechanics is a closed theory that is true wherever its concepts can be applied. This view was described as a sort of theoretical pluralism: there are certain domains of phenomena for which classical mechanics will forever provide the correct description (just as there are other domains of phenomena for which quantum mechanics is the final description). While Dirac also maintains a central role for classical mechanics, it is not as the final description of some particular domain. He sees classical mechanics as being very much an open theory—one that should continue to be developed, modified and extended. Unlike the contemporary orthodoxy, Dirac does not take the correspondence between classical and quantum mechanics to lie in some limiting procedure, nor to be confined to the borders of these theories. A reexamination of this 
historical debate between Heisenberg and Dirac not only deepens our understanding of their philosophical views, but also reveals that, when it comes to the relationship between classical and quantum mechanics, the currently favored positions of theory replacement and reductionism are not the only options. 


\section{Acknowledgements}

Earlier versions of this paper were presented at "Philosophical Issues in Physics: A Conference in Memory of James T. Cushing" held November 2002 at the University of Notre Dame and the 12th International Congress of Logic, Methodology and Philosophy of Science held August 2003 in Oviedo Spain; I am grateful to both sets of audience members for their feedback. I would like to thank Mélanie Frappier for sending me a copy of her paper and for stimulating discussions about Heisenberg. My gratitude also goes to Michael Leach of Harvard University's Physics Research Library for his assistance viewing materials from the Archive for the History of Quantum Physics. Last but not least this work was made possible by the generous support of the National Science Foundation, grant SES-0240328.

\section{References}

Beller, M. (1996). The Rhetoric of Antirealism and the Copenhagen Spirit. Philosophy of Science, 63, 183-204.

Beller, M. (1999). Quantum Dialogue: The Making of Revolution. Chicago: University of Chicago Press.

Bokulich, A. (forthcoming). Heisenberg Meets Kuhn: Closed Theories and Paradigms.

Bokulich, P. and A. Bokulich (forthcoming). Niels Bohr's Generalization of Classical Mechanics.

Cartwright, N. (1995). The Metaphysics of the Disunified World. In D. Hull, M. Forbes, and R. Burian (eds.), PSA 1994, vol. 2. East Lansing, MI: Philosophy of Science Association, 357-364.

Cartwright, N. (1999). The Dappled World: A Study of the Boundaries of Science. Cambridge: Cambridge University Press.

Chevalley, C. (1988). Physical Reality and Closed Theories in Werner Heisenberg's Early Papers. In D. Batens and J. P. van Bendegem (eds.) Theory and Experiment. Dordrecht: D. Reidel Publishing Co.

Cushing, J. T. (1990). Theory Construction and Selection in Modern Physics: The S Matrix. Cambridge: Cambridge University Press.

Darrigol, O. (1992). From c-Numbers to q-Numbers: The Classical Analogy in the History of Quantum Theory. Berkeley, CA: University of California Press.

Dirac, P.A.M. (1925). The Fundamental Equations of Quantum Mechanics. Proceedings of the Royal Society of London, Series A, 109, 642-653.

Dirac, P.A.M. (1938). Classical Theory of Radiating Electrons. Proceedings of the Royal Society of London, Series A, 167, 148-169. 
Dirac, P.A.M. (1945). On the Analogy Between Classical and Quantum Mechanics. Reviews of Modern Physics, 17, 195-199.

Dirac, P.A.M. (1951). The Relation of Classical to Quantum Mechanics. Proceedings of the Second Mathematical Congress, Vancouver, 1949. Toronto: University of Toronto Press.

Dirac, P.A.M. (1951). Is There an Aether? Nature, 168, 906-907.

Dirac, P.A.M. (1952). Is There an Aether? Nature, 169, 702.

Dirac, P.A.M. (1953). The Lorentz Transformation and Absolute Time. Physica, 19, 888896.

Dirac, P.A.M. (1958). The Principles of Quantum Mechanics (4th ed.). Oxford: Clarendon Press.

Dirac, P.A.M. (1963). The Evolution of the Physicist's Picture of Nature. Scientific American, 208(5), 45-53.

Dirac, P.A.M. (1970). Can Equations of Motion Be Used in High-Energy Physics? Physics Today, April, 29-31.

Dirac, P.A.M. (1971). The Development of Quantum Theory: J. Robert Oppenheimer Memorial Prize Acceptance Speech. New York: Gordon and Breach Science Publishers.

Dirac, P.A.M. (1974). Einstein and Bohr: The Great Controversy. Unpublished lecture. Paul A. M. Dirac Collection, Series 2, Box 29, Folder 4, Florida State University, Tallahassee, Florida, USA.

Dirac, P.A.M. (1982). The Early Years of Relativity. In G. Holton and Y. Elkana (eds.), Albert Einstein: Historical and Cultural Perspectives, Jerusalem Einstein Centennial Symposium 14-23 March 1979 (pp. 79-90). Princeton, NJ: Princeton University Press.

Dirac, P.A.M. (1987). The Inadequacies of Quantum Field Theory. In B. Kursunoglu and E. Wigner Reminiscences About a Great Physicist: P.A.M. Dirac. Cambridge: Cambridge UP.

Frappier, M. (forthcoming). Hilbert's Influence on Heisenberg's Closed Theories. Talk given at the History of Philosophy of Science Meeting held in Montréal, Canada, June of 2002.

Frisch, M. (2002). Non-Locality in Classical Electrodynamics. British Journal for the Philosophy of Science, 53, 1-19.

Heisenberg, W. (1926a). Mehrkörperproblem und Resonanz in der Quantummechanik. Zeitschrift für Physik, 38, 411-426. 
Heisenberg, W. (1926b). Quantenmechanik. Die Naturwissenschaften, 14, 899-994.

Heisenberg, W. (1934 [1979]). Recent Changes in the Foundations of Exact Science. In W. Heisenberg, Philosophical Problems of Quantum Physics (pp.11-26). Woodbridge, CT: Ox Bow Press.

Heisenberg, W. (1935 [1979]) Questions of Principle in Modern Physics. Lecture delivered at Vienna University on November 27, 1935. Published in W. Heisenberg, Philosophical Problems of Quantum Physics (pp.41-52). Woodbridge, CT: Ox Bow Press.

Heisenberg, W. (1942). Unpublished manuscript. Listed as Ordnung der Wirkleichkeit in W. Blum, H.-P. Dürr, and H. Rechenberg (eds.), Werner Heisenberg: Gesammelte Werke/ Collected Works, Series C, Part I. Munich: Piper, 1984. Translated from German into French with an introduction by C. Chevalley in Philosphie: Le Manuscrit de 1942. Paris: Éditions du Seuil, 1998.

Heisenberg, W. (1948 [1974]). The Notion of a "Closed Theory" in Modern Science. In W. Heisenberg Across The Frontiers (pp. 39-46). New York: Harper \& Row, Publishers. Originally published as Der Begriff 'Abgeschlossene Theorie' in der Modernen Naturwissenschaft. Dialectica, 2, 331-336.

Heisenberg, W. (1958). Physics and Philosophy: The Revolution in Modern Science. New York: Harper \& Row, Publishers.

Heisenberg, W. (1963, February 25). [Oral history interview of Werner Heisenberg by Thomas Kuhn]. Archive for the History of Quantum Physics, deposit at Harvard University, Cambridge, MA.

Heisenberg, W. (1963, February 27). [Oral history interview of Werner Heisenberg by Thomas Kuhn]. Archive for the History of Quantum Physics, deposit at Harvard University, Cambridge, MA.

Heisenberg, W. (1963, February 28). [Oral history interview of Werner Heisenberg by Thomas Kuhn]. Archive for the History of Quantum Physics, deposit at Harvard University, Cambridge, MA.

Heisenberg, W. (1970 [1974]). The End of Physics? In W. Heisenberg Across the Frontiers (pp. 184-191). New York: Harper \& Row, Publishers.

Heisenberg, W. (1971). Atomic Physics and Pragmatism (1929). In W. Heisenberg Physics and Beyond: Encounters and Conversations (pp. 93-102). New York: Harper \& Row, Publishers.

Heisenberg, W. (1972 [1983]). The Correctness-Criteria for Closed Theories in Physics. In W. Heisenberg Encounters with Einstein: And Other Essays on People, Places, and Particles. Princeton, NJ: Princeton University Press.

Infeld, L. (1952). Is There an Aether? Nature, 169, 702. 
Kragh, H. (1990). Dirac: A Scientific Biography. Cambridge: Cambridge University Press.

McAllister, J. (1996). Beauty and Revolution in Science. Ithaca, NY: Cornell University Press.

Sánchez-Ron, J. (1983). Quantum vs. Classical Physics: Some Historical Considerations on the Role Played by the "Principle of Correspondence" in the Development of Classical Physics. Fundamenta Scientiae, 4, 77-86.

Scheibe, E. (2001). Heisenberg's Concept of a Closed Theory. In B. Falkenburg (ed.), Between Rationalism and Empiricism: Selected Papers in the Philosophy of Physics. New York: Springer Verlag. 